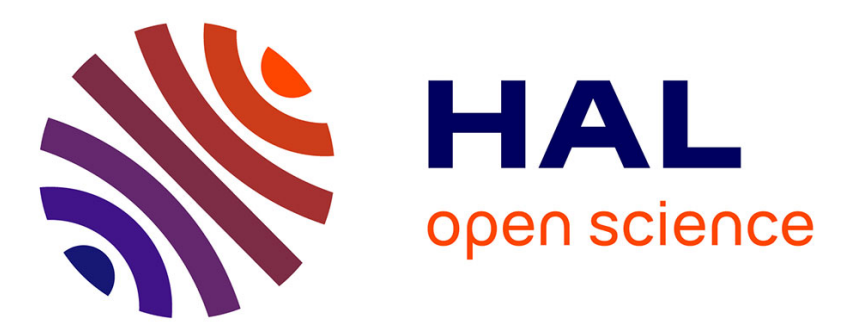

\title{
Mathematization of experts knowledge: example of part orientation in additive manufacturing
}

\author{
Mouhamadou Mansour Mbow, Christelle Grandvallet, Frédéric Vignat, \\ Philippe René Marin, Nicolas Perry, Franck Pourroy
}

\section{- To cite this version:}

Mouhamadou Mansour Mbow, Christelle Grandvallet, Frédéric Vignat, Philippe René Marin, Nicolas Perry, et al.. Mathematization of experts knowledge: example of part orientation in additive manufacturing. Journal of Intelligent Manufacturing, 2021, pp.1-19. 10.1007/s10845-020-01719-2 . hal-03152517

\section{HAL Id: hal-03152517 \\ https://hal.science/hal-03152517}

Submitted on 25 Feb 2021

HAL is a multi-disciplinary open access archive for the deposit and dissemination of scientific research documents, whether they are published or not. The documents may come from teaching and research institutions in France or abroad, or from public or private research centers.
L'archive ouverte pluridisciplinaire HAL, est destinée au dépôt et à la diffusion de documents scientifiques de niveau recherche, publiés ou non, émanant des établissements d'enseignement et de recherche français ou étrangers, des laboratoires publics ou privés. 


\title{
Mathematization of experts knowledge: example of part orientation in additive manufacturing
}

\author{
Mouhamadou Mansour Mbow ${ }^{1}\left[\right.$ ] Christelle Grandvallet ${ }^{1} \cdot$ Frederic Vignat $^{1} \cdot$ Philippe Rene Marin $^{1} \cdot$ Nicolas Perry $^{2}$. \\ Franck Pourroy ${ }^{1}$
}

\begin{abstract}
The use of expert knowledge by manufacturing companies to support everyday activities has become an emerging practice thanks to the new knowledge management tools. A big set of knowledge is available in the organizations but its profitable use to solve problems and assist decision making is still a challenge. This is the case of CAM operations or preprocessing steps for which various works have been led to involve experts' knowledge in the decision-making based on qualitative principles. However, so far, there is no methodology to the quantitative representation of that knowledge for more assistance. This paper introduces an approach for the conversion of knowledge into quantitative mathematical models. The main idea is to go from elicitation data in the form of action rules to simple unitary mathematical images; here desirability functions. The whole process carried out to extract the useful information that help building the desirability functions is exposed and different useful mathematical considerations are proposed. The resulting methodology identifies the categories of concepts in action rules and translate them into codified action rules. Then, through a mathematization process, the desirability functions are built. In short, this new approach allows evaluating the satisfaction level of the rules prescribed by the experts. As an illustration, the model is applied to action rules for CAM operations in additive manufacturing and more precisely to the definition of part orientation. This has shown the robustness of the methodology used and that it is possible to translate elicitation data into mathematical functions operable in computation algorithms.
\end{abstract}

Keywords Expert knowledge $\cdot$ Quantitative representation model $\cdot$ Desirability function $\cdot$ Action rule $\cdot$ Additive manufacturing $\cdot \mathrm{CAM} \cdot$ Part orientation

Mouhamadou Mansour Mbow

mouhamadou-mansour.mbow@g-scop.fr

Christelle Grandvallet

christelle.grandvallet@univ-grenoble-alpes.fr

Frederic Vignat

frederic.vignat@grenoble-inp.fr

Philippe Rene Marin

philippe.marin@grenoble-inp.fr

Nicolas Perry

nicolas.perry@u-bordeaux.fr

Franck Pourroy

franck.pourroy@grenoble-inp.fr

1 Univ. Grenoble Alpes, CNRS, Grenoble INP, G-SCOP, 38000 Grenoble, France

2 Univ. Bordeaux, CNRS, Arts et Métiers, I2M, Esplanade Arts Et Métiers, 33405 Talence, France

\section{Introduction}

Nowadays, companies are trying more and more to capitalize and organize their employees' knowledge in order to share it for better performance. This activity is referred to as knowledge management (KM) and has become a vital part of successful businesses as demonstrated by Tan and Wong (2015) through a work examining the effect of KM on manufacturing performance. Indeed, capturing, structuring and transmitting the workers knowledge, is a good way to assist activities like decision-making, problem solving, dynamic learning or strategic planning (Gupta et al. 1999). The knowledge management methodologies may vary from one company to another depending on their objective use of the created or captured knowledge (Davenport et al. 1998). Various authors worked on how leading KM would positively influence the competitiveness of manufacturing companies (Gunasekaran and 
Ngai 2007; de Pablos 2002; Singh et al. 2006; Paiva et al. 2002). Globally, it has been seen as an important element that maintains the sustainability and competitive advantage of an organization. In industries, the management of knowledge is critical to all processes of the value chain. Especially, the domain of product manufacturing requires intensive knowledge, from product design to production and quality inspection.

To manufacture a product implies to deliver at the right time and at an optimized cost in the required specifications, such as the right shape, dimensions, roughness, or material properties. To do so, multiple manufacturing strategies (for instance toolpaths) and parameters have to be set, which is the aim of CAM (Computer Aided Manufacturing) operations. Nevertheless, making the right choices requires a lot of knowledge and high expertise from the CAM engineer.

Regarding milling technologies, engineers rely on some commercial CAM software based on the following approach: feature recognition from the part CAD model; association of a process composed of one or several operations related to the identified features; setting of parameters related to these operations (Mourtzis et al. 2018). The automation of CAM operations mainly relies on expert systems based on if/then rules that make the CAM software hardly scalable. Moreover, relevant feature recognition often remains challenging in many situations (Albert 2001; Corney et al. 2005).

For more recent additive manufacturing technologies, CAM software are even less developed. They do not offer any assistance to the user for certain operations. On the other hand, some other operations that are sometimes fully automated (i.e. support generation) do not leave any control to the user.

Our research aims at defining various means of assistance for CAM operations. The target is to provide the CAM engineer with a decision assistant that would be based on the expert knowledge of the domain, and that would help him/her to make the right choices among numerous parameters to be set. In this context, the present paper proposes a structured approach for transforming manufacturing rules, gathered from a panel of experts and expressed in their natural language, into a quantitative mathematical model. This model will make it possible to assess in a simple way the extent to which the different manufacturing rules are satisfied when decisions are made about the CAM parameters.

In the remainder of this document, background and scope related to this research are presented in "Background and scope" section. Then, a case study of additive manufacturing part orientation that will be used for defining and illustrating our modelling approach is presented. The four main steps of this modelling approach are afterwards detailed in "Modeling approach" section. "Application to part orientation" section presents the modelling of some additive manufacturing rules and the application to a part geometry. Finally,
"Conclusion" section summarizes the results and provides concluding perspectives.

\section{Background and scope}

\section{Background}

The knowledge of CAM experts is the starting point this work. It is thus important to state what is meant by knowledge. Although some researchers consider that knowledge can be objectified and directly managed, for instance with information systems, the position that we adopt in this paper is more in line with Wilson (2002) who claims that knowledge "involves the mental processes of comprehension, understanding and learning that go on in the mind and only in the mind". In addition, Nonaka and Von Krogh (2009) make the distinction between explicit knowledge, which is "accessible through consciousness", and tacit knowledge that is more rooted in action, skills, movement, or senses. Knowledge is seen as a continuum between these two dimensions.

At the opposite side, information is defined as "everything outside the mind that can be manipulated in any way" (Wilson 2002). When available and formalized in documentation, information can be extracted for further processing (such as classification, storage, sharing or reuse).

Knowledge elicitation is "the process of collecting from a human source of knowledge, information that is thought to be relevant to that knowledge" (Cooke 1994). Various elicitation techniques, either based on observations, interviews or process tracing, have been widely used and studied in the literature (Milton 2007). These techniques make it possible for the experts to explain their knowledge, and hence to get some relevant information on this knowledge, that can afterwards be shared and managed. During knowledge elicitation, experts might become more conscious about certain parts of their knowledge. According to Nonaka and Takeuchi (1995), it is believed that some tacit knowledge moves towards the explicit dimension, making it possible to get information about this knowledge).

Knowledge structuring implies to classify it into, for instance, general and domain specific knowledge, concrete and abstract knowledge, formal and informal knowledge, conceptual and procedural knowledge (de Jong and Ferguson-Hessler 1996). While conceptual knowledge is valuable for the understanding of a specific domain, procedural knowledge is of prime importance in order to support action, and in particular to provide a CAM assistance.

Recent elicitation activities led in the manufacturing domain have enabled the formalization of experts' knowledge thanks to specific elicitation tools and techniques. When experimenting the use of an influence matrix in a 
collective debate, Grandvallet et al. (2017) managed to capture crucial knowledge about manufacturing practices, and classified it into different categories. Thereby, definitions, examples, influences, state rules were identified as four sub-categories of conceptual knowledge, and action rules that were part of procedural knowledge. The latter, action rules (ARs), are of primary interest in this work. Indeed, action rules can drive the process of setting multiple parameters. They are expressed in a natural language and integrate action verbs. In computational systems, they can be translated into different forms. For instance, they can take the form of a condition with if/then statement in expert systems (Zhou et al. 2019), they can be a basis for a reward system in machine learning algorithms, or they can be expressed in the form of a specific mathematical function.

Desirability functions prove to be suitable for the mathematical transformation of ARs. Desirability functions (DF) allow the conversion of different scales of quality measures into 0 to 1 objective functions (Trautmann and Weihs 2006). These functions were first introduced by Harrington, then, Derringer and Suich (1980) proposed a modification to show their ability to transform multi variate responses into only one single aggregated output. Different types of these functions are available in the literature (from the simplest to the most sophisticated) (Costa et al. 2011), and have been used combined with the response surface methodology or fuzzy logic to solve manufacturing parameter optimization problems (Datta et al. 2006; Aggarwal et al. 2008; Kim and Lin 2000; Singh et al. 2013; Al-Refaie et al. 2017). Figure 1 shows examples one sided and two sided desirability functions. Another advantage with this approach is the possibility to incorporate the decision maker preferences such as giving priority levels or weights to each response, for multiple response problems (Costa et al. 2011). Equation 1, shows an example using geometrical weighted mean proposed by Derringer and Suich (1980), where $w_{i}$ represent the weights given to each function.
$D=\left(d_{1}^{w 1} * d_{2}^{w 2} * d_{3}^{w 3} * \cdots * d_{i}^{w i}\right)$

$\sum w_{i}=1$

Three types of functions can be found, "the larger the better", "the nominal the best" or the "smaller the better". In this work, only the larger the best is used i.e. the most desirable case corresponds to a desirability index near the upper bound target $U$ and the less desirable corresponds to that near the lower bound $L$. The formulation of desirability functions has evolved through years with a variety of proposals. However, for the sake of simplicity, this study will consider the formulation proposed by Derringer and Suich (1980).

In the present context, DFs make it possible to quantify the satisfaction level of an action rule related to some variations of given variables. The use of desirability functions usually consists of identifying first the most important factors $(\hat{y})$ influencing the output response of the system of interest. Then, those are transcribed into desirability indices (d) (each value of the identified factor will have an image $d$ ranging from " 0 " to " 1 ") by experimental means or by using any expertise. In the present work, these factors or quantifiable variables $(\hat{y})$ are directly referred in the action rules either explicitly or implicitly. In the latter case, the variable is inferred from the fundamental relationship between the constitutive concepts of the AR. For this purpose, a relational analysis of the rules content must be led in order to extract the variable $\hat{y}$ and its threshold values allowing the mapping with desirability.

This short review of literature is summarized by Fig. 2 which shows the positioning of the paper.

On the right side of the diagram in Fig. 2, experts have more or less conscious knowledge that allow them to take relevant decisions regarding the choice of CAM parameters. Elicitation techniques make it possible to go to the information side since they provide information on this knowledge, and more especially in the form of action rules that can be
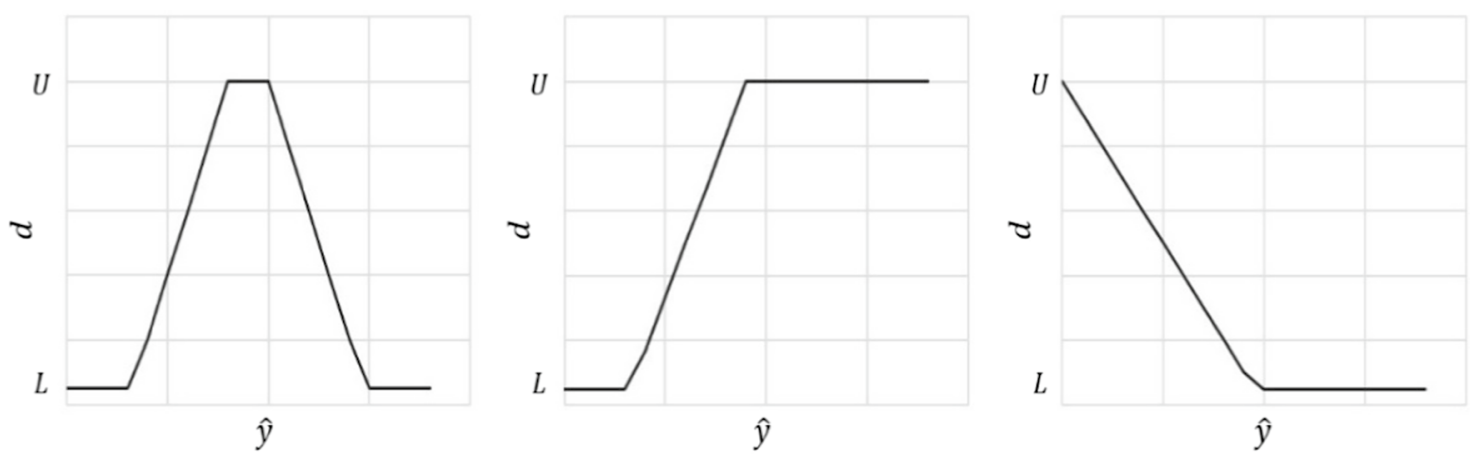

Fig. 1 Example of desirability functions 
Fig. 2 Knowledge transformation

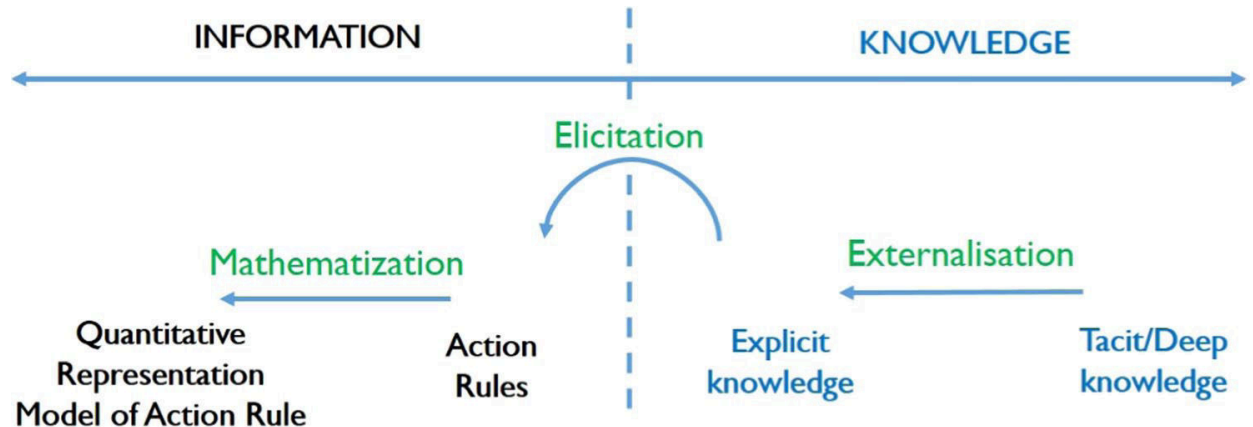

used to drive the CAM activities. These action rules can then be reworked through a mathematization process, using for instance desirability functions, in order to get quantitative representations. The term mathematization refers to the action of introducing principles and methods specific to the mathematical sciences into a field of knowledge that did not belong to it. It also refers to the act of giving a mathematical formulation to something (CNRTL - CNRS). It is an act of interpreting or expressing mathematically, or the state of being considered or explained mathematically (Collins dic.).

Thereafter, once integrated within a CAM environment, those quantitative representations will make it possible to evaluate easily and quickly the satisfaction or compliance level of each one of the action rules for a set of values of the CAM parameters. These evaluations will be useful for the engineers to make relevant decisions about these parameters.

Although it takes time to achieve this process, and skilled operators might consider more efficient to make decision based on their own knowledge, the proposed approach has the following advantages:

- The task of elicitation and mathematization of the company knowledge is only done once,

- The approach makes it possible to develop a companyspecific shared expertise,

- Decisions are justified based on measurable criteria.

\section{Scope of the paper}

The present paper aims at bridging the gap between the action rules, expressed in natural language, and a mathematical representation of these rules. The research question states as follows: which methodology based on desirability functions could be defined in order to get in a systematic way, quantitative representations of action rules that drive the CAM activities?

The proposed methodology is based on a study case about part orientation in AM. In this context, knowledge elicitation has been conducted with industry experts (Grandvallet et al. 2020). The resulting rules are used as the starting point of the work presented in this paper.

\section{Study case: part orientation in EBM manufacturing}

\section{Manufacturing process}

Electron Beam Melting (EBM) is one of the Powder Bed Fusion technologies (PBF) commonly used in Additive Manufacturing. The manufactured part is created layer by layer by melting metal powder with an electron beam. Figure 3 shows a typical PBF manufacturing process. Following the design step and preceding the manufacturing step, the CAM step encompasses various operations that aim at preparing the build file, including the settings of manufacturing parameters. For PBF technologies, this includes the selection of optimal build orientation, the creation of support structures for overhanging surfaces, the nesting on the build platform. But first and foremost, part orientation is one of the most important steps of CAM operations.

\section{Action rules for part orientation in EBM AM}

Part orientation is basically the setting of two angles that will define how the part should be oriented regarding the building plate of the machine (start plate). Nevertheless, it remains a complex and critical activity as it highly affects the quality, cost, production and finishing time of the part.

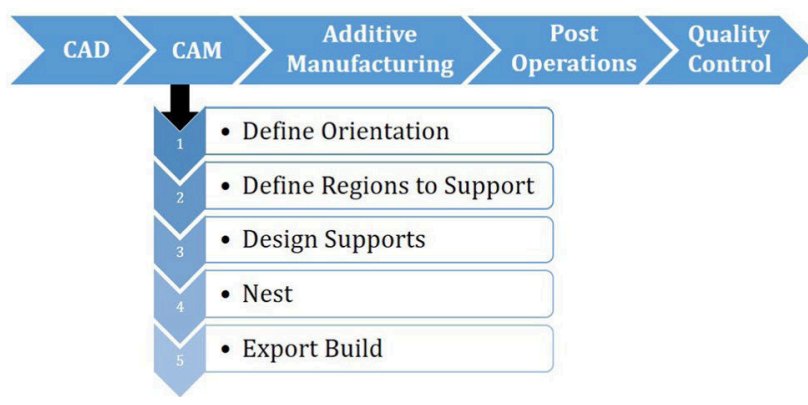

Fig. 3 Example of additive manufacturing process for powder bed fusion (PBF) 
Usually, operators arbitrarily orient parts without being able to explain why they chose a given orientation. Various authors in the literature have investigated the computation of part orientation (Frank and Fadel 1995; Delfs et al. 2016; Pandey et al. 2007; Das et al. 2015; Rattanawong et al. 2001; Zhang et al. 2017; Qin et al. 2019). Typically, the computation of optimal orientation usually consists in selecting a set of criteria to fulfill, then evaluating those on geometries of interest. The most encountered aspects consist of minimizing: the support volume or area, the build time, the build height, the material consumption, the projected area and the distortions.

Today more and more commercial software are used to compute these criteria on parts as indicated in Table 1 . These tools are globally using physical parameters of different scales (e.g. support volume, part height, etc.) that cannot be compared to each other. Most of them propose optimum solutions computed by assigning weights to the aforementioned dimensional physical parameters. These weights remain the only values on which the operator can intervene, as the algorithms used by the commercial tools are black boxes. Beyond the criteria computation, some of the tools allow the definition of surface category (3DXpert, Netfabb and Magics). Only one software (Amphyon) allows a heatmap presentation of the criteria values over the possible orientations to support decision-making. Moreover, the criteria defined by the software editor cannot be evolving with the expertise of the company; they do not offer to experts the possibility to add new orientation rules or criteria.

As mentioned in "Scope of the paper" section, the originality of the approach proposed in this paper consists in starting from expert knowledge for building a set of rules and dimensionless criteria applied to specific part entities, so as to support decision in part orientation.

In an industrial context, Grandvallet et al. (2020) used the LIT (Limited Information Task) technique in order to capture main concepts involved in the execution of part orientation. Eight action rules were constructed from statements of industry experts working on powder-based additive manufacturing. Six of them have been more deeply studied for mathematization. They are listed and explained hereafter:

(a) Minimize part shadow on start plate: the projected area of the part on the build platform (start plate) must be minimal to allow the maximum nesting (Chergui et al. 2018; Wang et al. 2019). The extent of the projected area varies with the orientation of the geometry.

(b) Minimize total overhanging non-machined surfaces: overhanging surfaces need support structures during the manufacturing process to get the surface anchored and prevented from distortion or warping (Hussein et al. 2013). When support structures are used, their removal leaves marks that usually need finishing or pol-

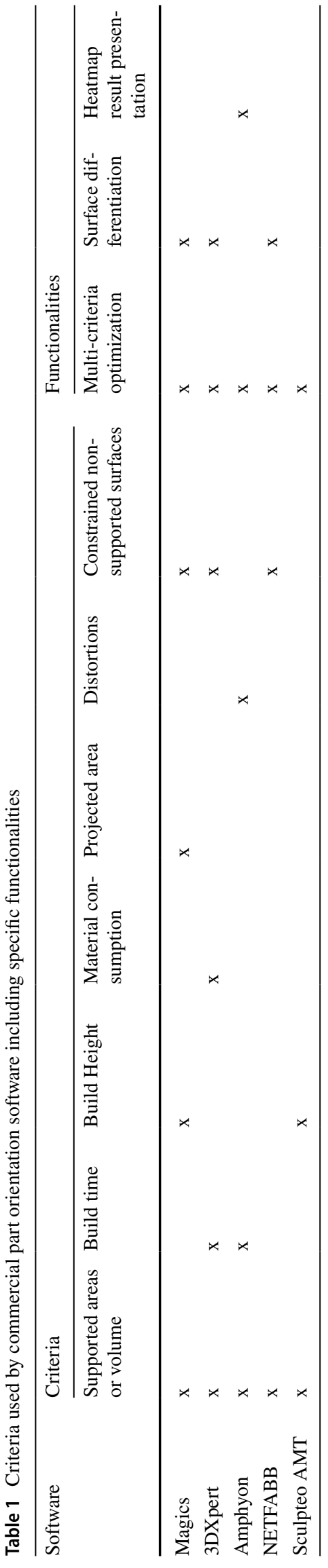




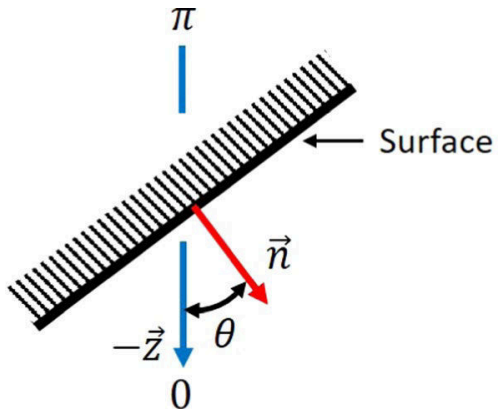

Fig. 4 Illustration of overhanging surface

ishing. This rule intends to minimize the marks (thus, overhanging surfaces) on surfaces for which machining will not be performed. An overhanging surface is characterized by its normal vector angle with the negative vertical as shown in Fig. $4(\theta=(\vec{n},-\vec{z}))$. Usually, support structures are only required for surface angles less than a specified value. This value depends on the technology, the material and the machine.

(c) Orient part priority surfaces (PPS) close to vertical: priority surfaces constitute the most important surfaces of a given part. In PBF, surfaces oriented vertically have better quality, thus priority surfaces are oriented as close as possible to the vertical.

(d) Orient machining datum surfaces (MDS) out of horizontal: machining datum surfaces allow the positioning of the part for post processing. If they are positioned horizontally (close to $0^{\circ}$ or close to $180^{\circ}$ ), they might exhibit geometrical distortions.

(e) Minimize shape deformation risks: parts made with PBF technologies are usually subject to deformations linked with the solidification and cooling process of the fused metal layers. According to experts, if a certain number of long layers are stacked, there may be risk of deformation. Changing the orientation of a part modifies the configuration of the layers through a different slicing. Therefore, this issue is to date mainly solved by finding the convenient building orientation. Note that only shrinkage defect (Vo et al. 2018; Ghaoui et al. 2020) is concerned with this rule.

(f) Avoid support structures and support removal difficulty on surfaces with potential support difficult to remove (SSDR): this rule suggests to take into account the support removal difficulty. In PBF, a tool is used to remove the support structures after manufacturing, for this reason, the accessibility needs to be considered. Here, by making the assumption that the support is difficult to remove when the surface in question has another one in front of it, the removability depends on the space between the two surfaces. A surface is therefore quali- fied as a SSDR when its accessibility is limited by surrounding surfaces.

Usually, action rules can easily be interpreted or even completed by human reasoning. However, to do so with computational systems, mathematical formal models have to be established. To this end, the next section presents the proposed modeling approach that allows interpreting the action rules content into desirability functions.

\section{Modeling approach}

The proposed approach consists in translating action rules expressed in natural language into fuzzy functions. The main aim is to provide a mathematical image as close as possible to the objective of a given action rule. To achieve this, desirability function based modeling is used. This modeling approach has got the advantage of normalizing variables of diverse natures into dimensionless values between zero and one.

Figure 5 summarizes the general steps of the methodology. The first step (1) is to codify the action rule in order to put it in the form of an objective (if necessary) using a common grammar. The second step proceeds to a mathematization of the action rule by performing the following steps: (2) Define

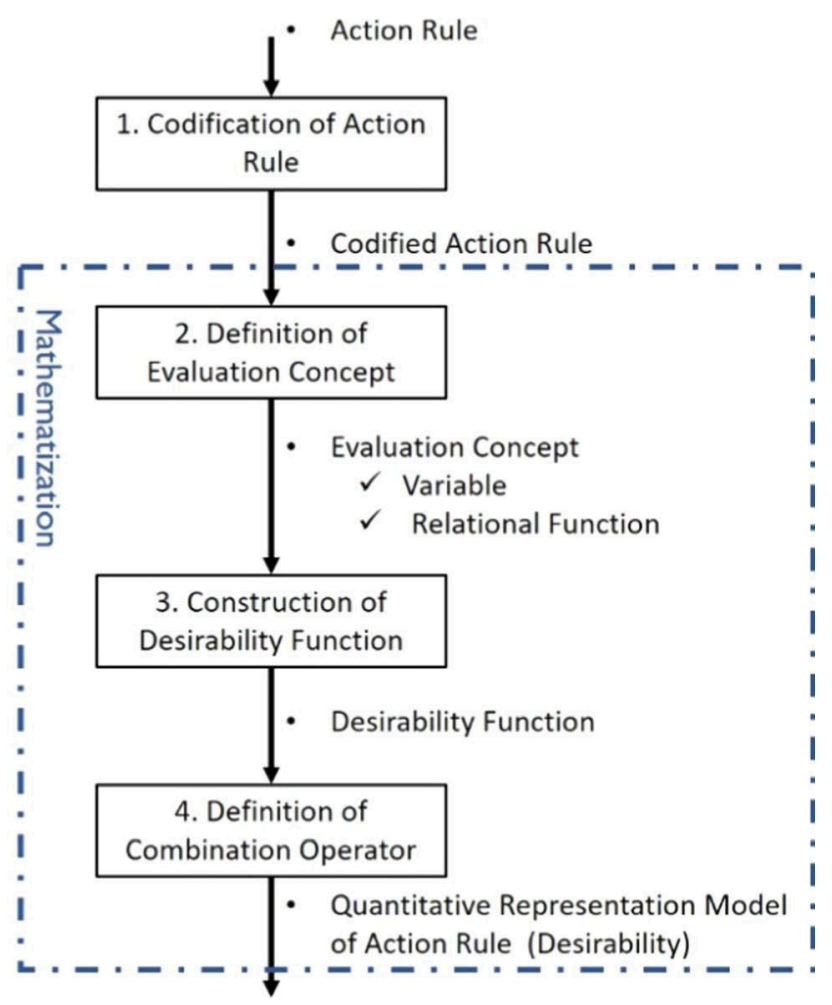

Fig. 5 Proposed modeling steps 
the evaluation concept, (3) Construct the desirability function, (4) Define a combination operator. In (2) a relational analysis of AR content is carried out to find out the quantifiable variables $\hat{y}$ and their related evolution laws that will be used for the construction of desirability functions in (3). The final step (4) consists of defining how the overall desirability is calculated on the whole part. It has to be noted that the experts may intervene in the whole modeling process.

\section{Codification of action rules}

Action rules captured from expertise are expressed in different forms with different grammatical structures. This diversity makes it difficult to define and handle a general modeling process. For that reason, this work proposes to codify the ARs in the form of objectives using a common grammar made of words and classified in different categories of concepts related with the domain of interest.

For additive manufacturing, Kim et al. (2019) recently proposed a classification that includes these categories: Design Features, Manufacturing Features, Geometry Parameter, Material Parameter, Process Parameter and Feature Constraint. The Design Features are linked with the part topology (e.g. cylinder, spheres, surfaces, holes...), while the Manufacturing Features are those related to additive manufacturing itself (e.g. support structures, powder, layer ...). The Geometry Parameters correspond to characteristics that a Design Feature includes; for instance, a cylinder might have the following feature characteristics: height, diameter, area and orientation.

The proposition is not completely in line with the rules described "Action rules for part orientation in EBM AM" section. In fact, industrial practitioners differentiate the part geometric entities (shape entities) and gives them different attributes. Among those are the post-machined surfaces, the priority surfaces and the machining datum surfaces. A given surface can have multiple attributes (e.g. a non-machined surface can also be a priority surface). Action rules then apply to a subset of the part shape entities identified by their attributes.

Finally, the following categories have been identified: Shape entity, Attribute, Evaluation Concept and Action (Fig. 6). Given an action rule, its composition is such that the action applies to one or more evaluation concepts. Each evaluation concept is associated to shape entities identified by the given attribute.
Part

This concept clusters the different classes of information related to the geometry in question. The class Shape is defined by the distinct entities (volume, surfaces, edges and vertices) constituting the part's topology. As a complement, the class Specifications (corresponding to the global product specifications) indicates the extrinsic and intrinsic characteristics of the Shape entities. For instance, this class provides information about the roughness or the tolerances on the dimensions of a surface or between surfaces. The class Attributes is used to classify the Shape entities according to their expected quality or post processing need (e.g. machined surfaces). Figure $7 \mathrm{a}$ shows a summary of a part composition. A non-exhaustive list of attributes including hole, non-machined, machining datum and priority are defined based on the ARs formulated by experts. It has to be noted that the attributes may or may not exist in the action rule. They point to the involved Shape entities as illustrated in Fig. 7a by the dashed lines.

\section{Evaluation concept}

The evaluation concepts are used for assessing the impact of an action on a part of interest. Evaluation concepts are the pivotal base of the decision-making through an AR since they allow evaluating and understanding the consequences of an action onto the final requirements namely, the quality, cost and processing time. In the AR, each evaluation concept is linked to a shape entity by quantifiable variables (discussed in "Identify the variable $\hat{y}$ linking the shape and the evaluation concept" section). The identified evaluation concepts for the list of rules given in the "Action rules for part orientation in EBM AM" section are reported in Fig. $7 \mathrm{~b}$.

\section{Action}

The Actions are introduced to normalize the ARs in order to explicitly express them as objectives by using the following word units: minimize, maximize, avoid or require. They make it possible to transform an action rule into an objective to reach. Minimize and maximize terms are used when intermediary cases are accepted and, avoid and require are used when the non-acceptable cases (zero desirability) cannot be compensated (i.e. orientation with non-acceptable local situation has to be excluded).
Fig. 6 Example of action rule's concepts codification

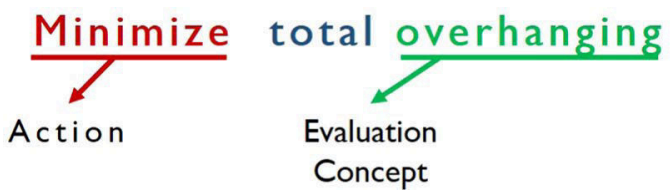

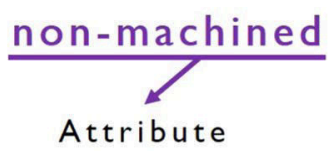

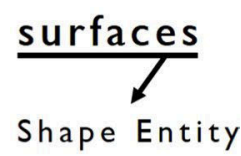



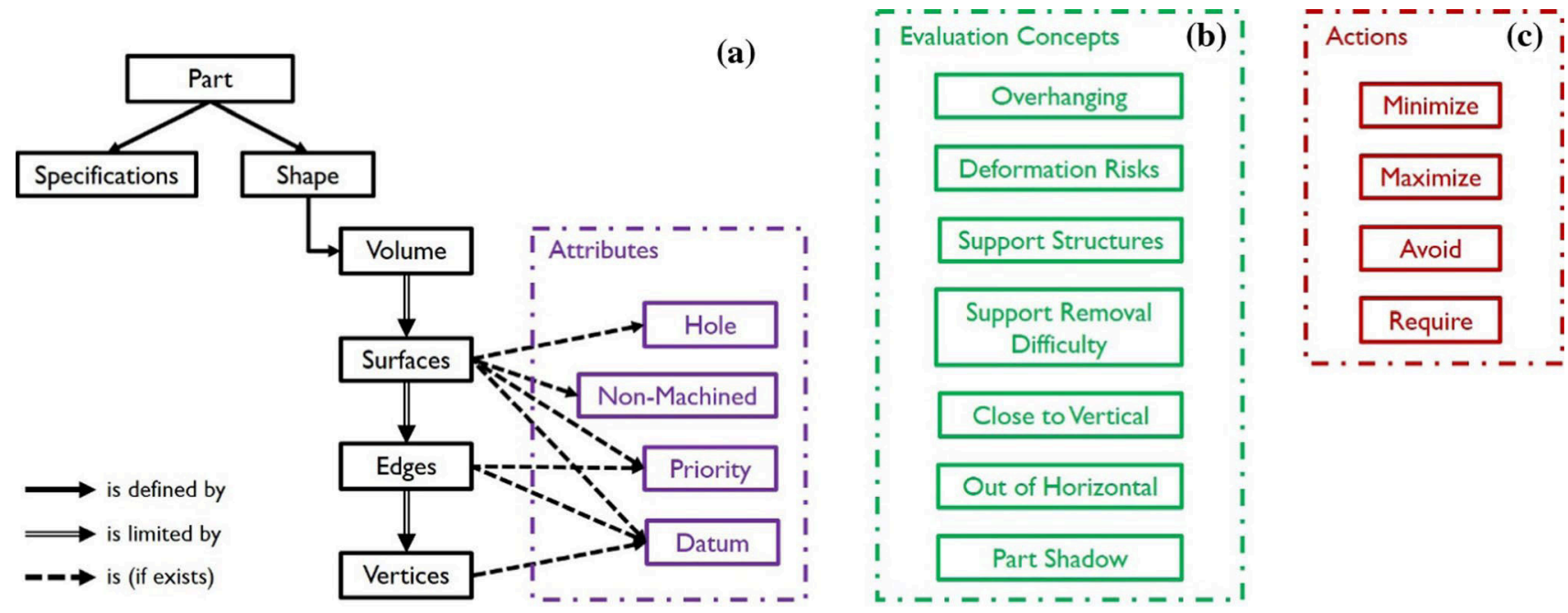

Fig. 7 Categories of concepts: a part classification; $\mathbf{b}$ evaluation concepts; $\mathbf{c}$ actions

\section{Codification}

Each action rule implicitly includes an objective to complete. The codification intends to explicitly show the objective of the action rule by replacing its action verb with one of the Actions defined in Fig. 7c. For instance, the rule "Orient machining datum surfaces (MDS) out of horizontal" seeks to get the maximum MDS possible out of horizontal position. Therefore, the codified version of this example could be either "Maximize MDS out of horizontal" or "Require MDS out of horizontal" depending on the wanted severity. It should be noted that the codification step is not always necessary because action rules are sometimes directly expressed in the form of objectives. For instance, in the aforementioned list, rules (a), (b), (e) and (f) are already codified.

\section{Definition of the evaluation concept}

In the literal expression of action rules, there are implicit laws involving the necessary understanding of the interrelations linking the concepts. Beissner et al. (1994) define the relation between the concepts as structural knowledge. The process of building the structural knowledge might require the preliminary scientific comprehension of interactions between the involved parameters and the intervention of experts to establish threshold values.

The idea in this step is to perform a mathematization of the action rules to represent the relationship between the concepts by following three main steps. As discussed in its definition, an evaluation concept is linked to the specified shape entity by quantifiable variables. Therefore, the variable $\hat{y}$ linking the shape entity and the evaluation concept is first identified by experts; second, the way to measure $\hat{y}$ on the part is determined. Finally, the calculation of the evaluation concept evolution according to the variable $\hat{y}$ is established. The expected outputs are $\hat{y}$, the way to measure it and the function linking $\hat{y}$ with the evaluation concept (Fig. 8).

\section{Identify the variable $\hat{y}$ linking the shape and the evaluation concept}

This process involves the manufacturing experts and CAD experts' knowledge. The aim is to identify the evaluation concept input variables $(\hat{y})$. These variables are selected with CAD experts as far as they have to be measured from the shape entities. In fact, each shape entity has some characteristic parameters that can be used as variables. For instance for a surface, the angle, the length of its perimeter, the area or the projected area, etc. can be identified. The parameter among those, that fits more with the evaluation concept will be used as the variable $\hat{y}$. Different opinions might arise among experts for the identification of $\hat{y}$. The part shadow onto the machine plate for which different types of measures have been identified is one illustration (refer to Fig. 9). The

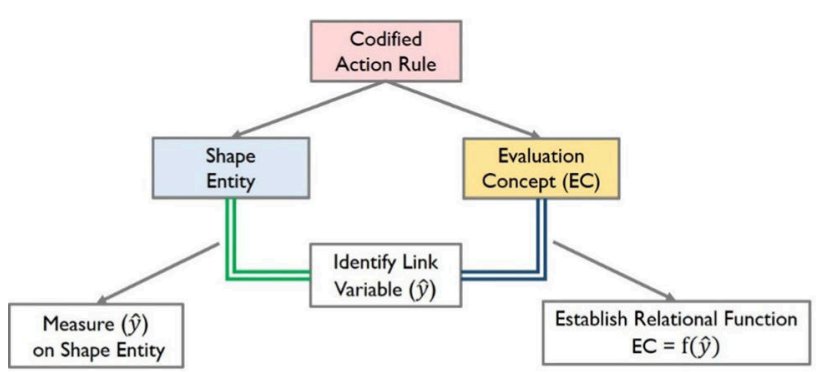

Fig. 8 Relationship between concepts, illustration of variable linking a shape entity and an evaluation concept 

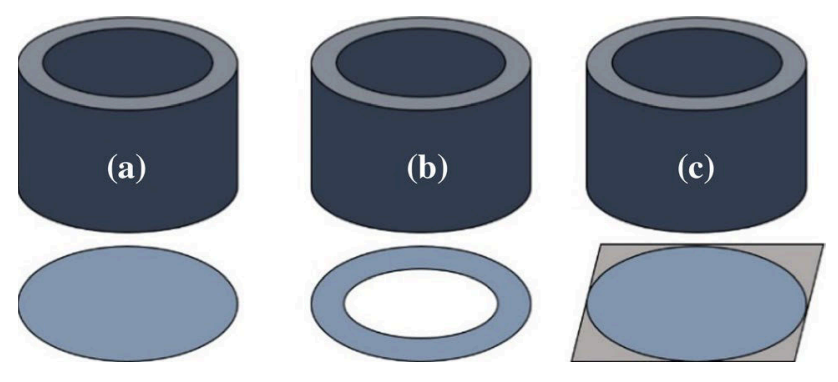

Fig. 9 Illustration of part shadow measurement possibilities

measurement of part shadow can be the area of the projection, the maximum dimension of the projection, and the projection itself can be the silhouette (Fig. 9a), the contour (Fig. 9b) or the bounding box (Fig. 9c). An iterative discussion with experts helped to finally converged on the solution (b) of Fig. 9. Indeed, in PBF it can be foreseen to put another part inside the hole of the shadow in order to save place.

\section{Determine how to measure the variable $\hat{y}$ on the part}

According to the part's involved entity, the way to measure the variable might differ. For some cases, a specific discretization method for local evaluation is required. For instance, to find the local angle $\hat{y}$ of a complex surface by its normal, it is necessary to tessellate the latter to transform it into triangular flat elemental surfaces. Helpful geometrical operations on shape entities for the evaluation of desirability are reported in Table 2.

\section{Find out how to calculate the evaluation concept according to the variable $(\hat{y})$}

This establishes the relationship between the $\hat{y}$ and the evaluation concept. This analysis is one of the most important steps that allow going from a conceptual view to a process view of the ARs. The expected output for each identified variable is a relational function (RF) showing the evolution of the evaluation concept according to $\hat{y}$.

For instance, given the codified action rule depicted in Fig. 6, a codification according to the discussed categories firstly allows identifying the concepts on which the analysis is carried out. The attribute non-machined indicates that the AR does not apply to all of the surfaces of the geometry of interest. For the identification of the linking variable $(\hat{y})$, the response is the inclination angle $(\theta)$ of the surfaces as the law of evolution of overhang (evaluation concept) depends on the surface orientation (explanation in "Action rules for part orientation in EBM AM" section). A tessellation is performed to measure the elementary $\theta$ of the involved surfaces. Then, the evolution of the overhang according to the inclination angle can be inferred in Fig. 10. The threshold values $\theta_{1}$ and $\theta_{2}$ must be indicated by the experts.

Thereafter, the desirability functions are constructed based on the established structural knowledge. As shown in Fig. 10, the RFs are mapped between 0 and 1, but for some evaluation concepts, a fuzzification is necessary (refer to "Fuzzification" section). In case of more than one evaluation concept, the mathematical considerations in "Mathematical combination of various evaluation concepts" section are used to carry out logical combinations.

Fuzzification Evaluation concepts can be of different types; they can be either non-quantifiable notions (e.g. deformation risks) or quantifiable notions (e.g. part shadow). In the first case, the relational diagram is built based on the importance degree of the evaluation concept according to the variable. In the second case, the relation is dictated by the maximum and minimum values of the quantifiable evaluation concept.

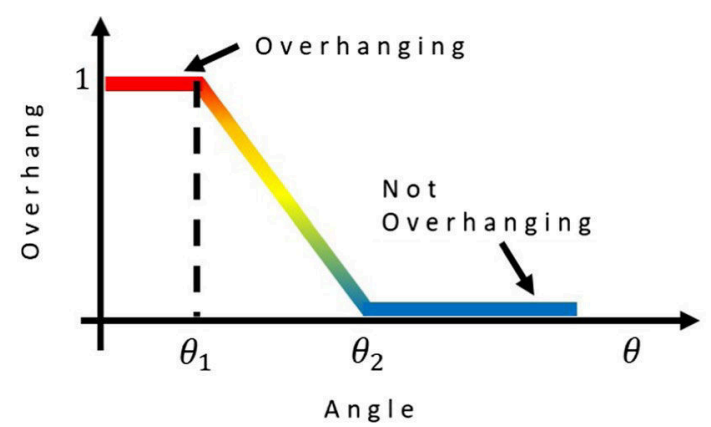

Fig. 10 Relational function between the evaluation concept (overhang) and the linking variable $(\hat{y}=\theta)$
Table 2 Geometrical operation for variable assessment

\begin{tabular}{llll}
\hline Shape entity & Request & Geometrical operation & Discretization \\
\hline Volume & Shadow area or length & Projection & - \\
& Size (length, width, height) & Bound-box & - \\
& Layer area & Contour filling (triangles, pixels) & Slicing \\
& Layer max/min length & Multi-directional hatching & Slicing \\
Surface & Shadow area or length & Projection & - \\
& Surface angle & Normal vector mapping & Tessellation \\
& Surface area & Triangular filling & Tessellation \\
\hline
\end{tabular}


For these reasons, a fuzzification is led to scale the evaluation concepts between 0 and 1. This consists in mapping their maximum and minimum possible values with values defined in the interval $[0,1]$.

For a variable $\hat{y}$, there is a function associating to each of its values a real $m \in[0,1]$ defined by:

$m=f(\hat{y})$

This definition describes the relation between the evaluation concept and the variable linking it to the involved shape entity of the part. For instance, if the shadow area is considered, the maximum and minimum values are not limited between zero and one. Therefore, the fuzzification is done such that the maximum possible shadow area for this part is assigned the value 1 and the minimum the value 0 .

Mathematical combination of various evaluation concepts The previous illustration (Fig. 10) shows a simple case for which, there is a single variable, however, in some cases, more than one variable can exist depending on the number of evaluation concepts. Logical operations such as "and" and "or" bind the latter; for instance, the rule (f) in "Action rules for part orientation in EBM AM" section, for which, there is an "and" operator. Hence, some mathematical notions are introduced to make possible the combination of multiple desirability functions. The properties used here are the generalization of properties used in fuzzy logic theory introduced by Zadeh (1965). A few definitions such as the negation of a RF, the intersection or union of multiple RFs are given in "Appendices" section.

\section{Construction of desirability functions}

As described in the Introduction, the desirability functions make it possible to translate any variable into normalized positive values in the $[0,1]$ interval. For the CAM operations, the purpose of using expert knowledge is to compare different scenarios of parameter combination for the sake of quality, cost or time optimization (main drivers for experts to define evaluation concepts). Thus, here, the desirability approach makes it possible to see any of the experts' rules in the same map of comparable dimensionless values.

The desirability function of each action rule is constructed based on the relational function from the mathematization and its relative action. For minimize and avoid, the corresponding desirability function is the opposite of the relational function (Eq. 4). Then, for maximize and require actions, the desirability function will be equivalent to the relational function (Eq. 5).

$D F=\overline{R F}=1-f(\hat{y})$
$D F=R F=f(\hat{y})$

In the example of Fig. 10, the variable linking the part involved shape entity (surface) and the evaluation concept (overhanging criteria) is the angle $(\theta)$ between the surface and the built direction. This angle measures the surface orientation relative to the $\mathrm{Z}$ build direction (see Fig. 4). The need of support depends on this angle. In fact supports are needed for overhanging surface i.e. with low $\theta$ values. The unit relational function describes when a surface is overhanging and needs support i.e. the angle $\theta$ is less than $\theta_{1}$ and when the surface is progressively not overhanging i.e. $\theta$ tending to be greater than $\theta_{2}$. To get the desirability function representing the action rule, the related action "minimize" is used. Owing to the fact that this action rule prescribes to minimize the overhanging non-machined surfaces, the situation in which those are overhanging is not desirable and the opposite situation is desirable. Therefore, the desirability function in Fig. 11 is obtained by inverting the relational function in Fig. 10.

This function associates to each value of the variable $\theta$ of the considered surface a satisfaction value ranging from 0 to 1 (the best values being those near 1).

\section{Definition of combination operator}

Analyzing various desirability values simultaneously is not a trivial manual task. When the surfaces of a part are considered for instance, the involved AR satisfaction level has to be discretely evaluated on each surface. Specially, if discretization has been performed on the shape, it becomes necessary to combine the local desirability values. Figure 12 shows an example of tessellated part made of over 16,000 triangles; this number of entity is impossible to handle manually when comparing scenarios. For such reasons, it is better combining the local desirability values to get a composite one by using an adequate averaging operator.

To evaluate the global desirability of a given geometry, the desirability is first calculated locally on each of the $n$ elemental facets obtained by tessellation (Fig. 12), then, calculated for the whole geometry itself by averaging on

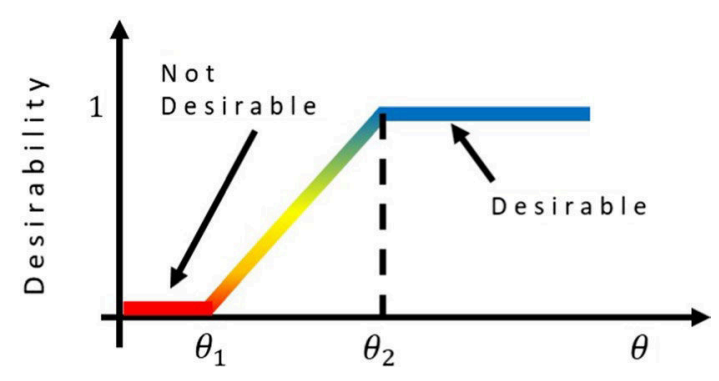

Fig. 11 Desirability function 


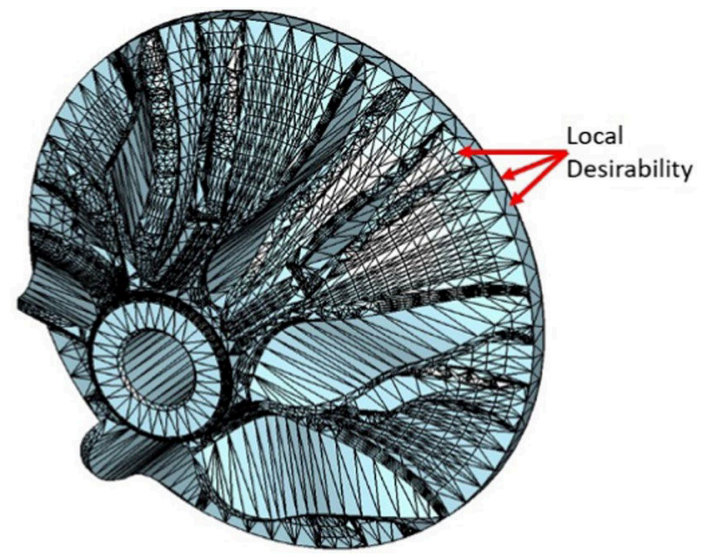

Fig. 12 Tessellated geometry (n Facets)

$n$ local values. There are various averaging possibilities in mathematics, but, as the $\mathrm{n}$ facets of the geometry might not have the same significance, it is necessary to use weighted averages. In this study, the generalized Hölder weighted power mean (Bullen et al. 2003) is suggested. The two relations in Eqs. 6 and $7\left(r_{1}, \ldots, r_{i}, \ldots, r_{n}\right.$ are positive weights $\in[0,1]$ for which $\sum r_{i}=1$, and $d_{1}, \ldots, d_{i}, \ldots, d_{n}$ are positive real numbers representing local desirability) are considered.

$D=\prod_{i=1}^{n} d_{i}^{r_{i}}$

$D=\sum_{i=1}^{n} r_{i} d_{i}$

The result in Eq. 6 is by definition the geometric mean weighted by the reals $r_{i}$. Similarly, the Eq. 7 indicates the weighted arithmetic mean which has been demonstrated to be greater than the geometric one (Qi et al. 2000). When the weights $r_{i}$ are equal, Eqs. 6 and 7 become equivalent to classic means. Here, the $r_{i}$ are linked with a characteristic parameter of the involved shape element. They can for example be relative surface area: $r_{i}=\frac{A_{i}}{A_{\text {total }}}$. This would give more importance to big surfaces at the expense of the smaller ones. It has been noticed that experts commonly define weights with facet area. Exploring different possibilities is part of future works.

The choice of the averaging operation depends on the Action prescribed in the AR. As aforementioned, the categories minimize and maximize show that intermediary values are accepted, thus they will use a compensatory operation i.e. the weighted arithmetic average as it refers to a sum.

On the other hand, avoid and require categories do not accept the zero desirability cases and will use the geometric weighted average (due to the product, the compensation effects are relatively low). This means that even if only one of the elemental entities $i$ has a null desirability, the whole geometry of $n$ entities will have an aggregated weak desirability regardless the other values.

Note that the discretization parameters affect the combined values very slightly. For the triangulation case, different parameters have been tested, but the combined desirability remained constant regardless the number of triangle. For complex parts with massive number of surfaces, the computation time might be long; its minimization should be considered in future developments.

\section{Application to part orientation}

\section{Modeling the action rules}

This part presents an example of knowledge modeling process about one of the most important CAM steps in additive manufacturing, which is the orientation of parts in the machine's build space. As suggested by the modeling approach, the first step is the codification of all action rules. Then, from a relational analysis of the content, a variable linking the part and the evaluation concept is inferred. Finally, the desirability functions are built and for each one, the corresponding evaluation formulae or averaging operator is provided. Results are summarized in Table 3.

Action rule (f) is a special case as it has two evaluation concepts thus, two link variables. The desirability of a SSDR is given by the intersection of the support structures requirement $(A)$ and support removal difficulty existence $(B)$. A mathematical formulation of the rule and its equivalent can be written this way:

\section{Avoid $A$ and $B$ on SSDR $=$ Require $\overline{A \text { and } B}$ on SSRD}

The corresponding relational functions are depicted in Fig. 13.

By applying the De Morgan law given in Eq. 14, it follows:

Require $\overline{A \text { and } B}$ on SSRD $=$ Require $\bar{A}$ or $\bar{B}$ on SSDR

Then, the equivalent desirability function $f_{c}$ of the rule will be given by the maximum between $\left(1-f_{A}\right)$ and $\left(1-f_{B}\right)$ (Fig. 14).

Experts must indicate the threshold values in all of the desirability functions. Note that if the threshold values are equal (e.g. $d_{1}=d_{2}$ ), the functions will become binary functions that can only take the values 0 or 1 .

\section{Example of application to a geometry}

\section{Problem description}

Consider the bracket depicted in Fig. 15; the aim is to determine an optimal orientation around the $\mathrm{X}$ and $\mathrm{Y}$ axes with 
Table 3 Modeled AM CAM action rules

a. Minimize part shadow on start plate

Codification: not required

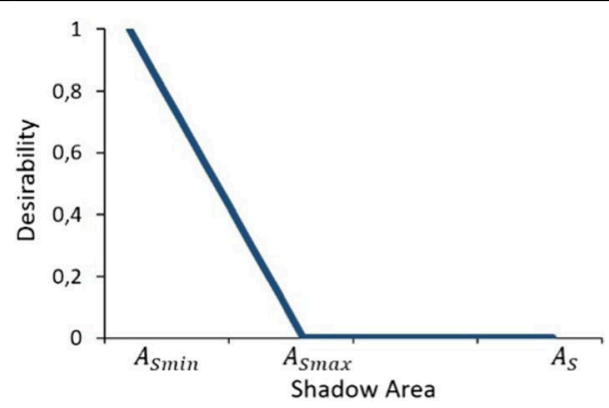

Shape entities: volume

Evaluation concept: shadow

Link variable: shadow area $\left(A_{S}\right)$

Geometrical Operation: orthogonal projection

Averaging Operator: none

b. Minimize total overhanging non-machined surfaces

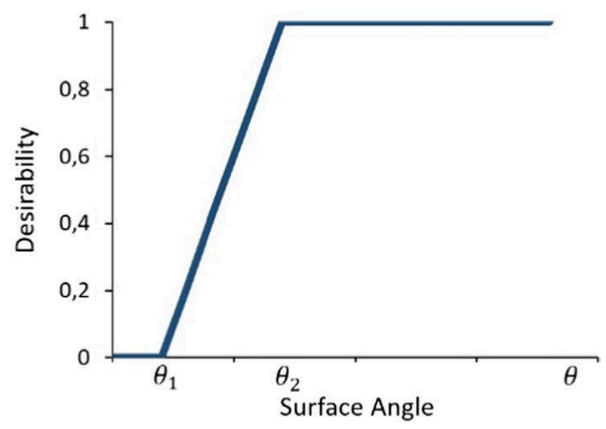

Codification: not required

Shape entities: (non-machined) surfaces

Evaluation concept: overhang

Link variable: surface angle $(\theta)$

Geometrical Operation: tessellation

Averaging Operator: arithmetic mean weighted by relative area

c. Orient part priority surfaces close to vertical

Codification: Maximize part priority surfaces close to vertical

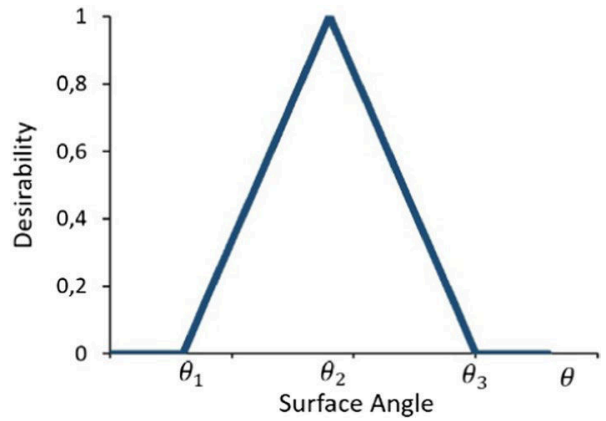

Shape entities: priority surfaces

Evaluation concept: close to vertical

Link variable: surface angle $(\theta)$

Geometrical Operation: tessellation

Averaging Operator: arithmetic mean weighted by relative area 
Table 3 (continued)

d. Orient machining datum surfaces out of horizontal

Codification: Require machining datum surfaces out of horizontal

Shape entities: machining datum surfaces

Evaluation concept: out of horizontal

Link variable: surface angle $(\theta)$

Geometrical Operation: tessellation

Averaging Operator: geometric mean weighted by relative area

e. Minimize shape deformation risks

Codification: not required

Shape entities: volume

Evaluation concept: deformation risks

Link variable: number of stacked long layers $(n)$

Geometrical Operation: slicing

Averaging Operator: weighted arithmetic mean

f. Avoid support structures and support removal difficulty on surfaces with potential support difficult to remove

Codification: not required

Shape entities: surfaces with potential support difficult to remove

Evaluation concepts: support structures, support removal difficulty

Link variables: surface angle $(\theta)$, distance face/face $(d)$

Geometrical Operation: tessellation

Averaging Operator: geometric mean weighted by relative area
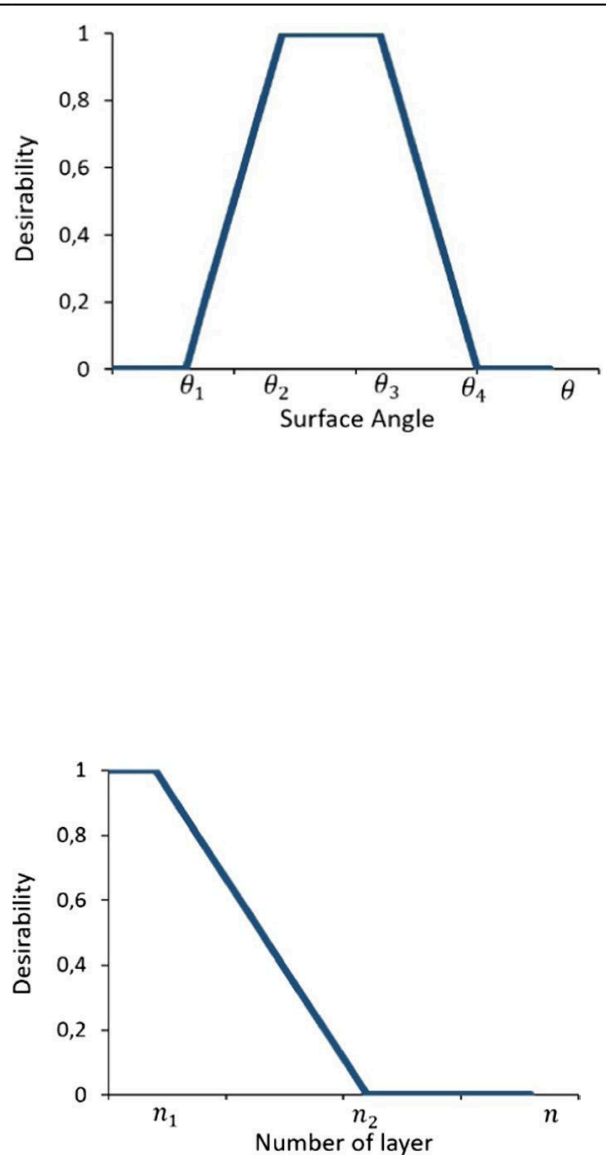

Multiple desirability function case developed below respect of the action rules (a), (b), (c), (d), (e) and (f) from Table 3. Their corresponding threshold values are given in Table 4 . The threshold values are proposed by the experts according to their machine capabilities. Some of them depend on the part geometry. For instance, for the part shadow rule, the thresholds are maximum $\left(\mathrm{A}_{\max }\right)$ and minimum $\left(A_{\text {min }}\right)$ area of part shadow (for the given part).

The green surfaces are post machined, thus, no matter if they have support structures. In fact, the machining price is already expected on those surfaces, that's why, the experts state that it's better putting support structures on them to save the non-machined surfaces (which include all of the other surfaces). Priority and datum surfaces are highlighted in red and yellow respectively. The surfaces with potential support difficult to remove are computed by a dedicated algorithm, since their manual selection would be difficult to achieve. 
The evaluation of the ARs on the geometry is made using a $\mathrm{C}++$ program based on Opencascade libraries (for geometry processing). This tool makes it possible to easily add Attributes to the shape entities. The rotations around $\mathrm{X}$-axis ( $\mathrm{Rx}$ ) range from $0^{\circ}$ to $180^{\circ}$ and those around $\mathrm{Y}$-axis (Ry) range from $0^{\circ}$ to $360^{\circ}$. The results displayed in the form of response surfaces.

\section{Results}

Figure 16 shows for action rule (a) a total of 648 different desirability values of the bracket's orientations. The effect of the type of action (e.g. minimize) can be noticed on some of the results for which, aggregation on the whole part has been carried out (refer to "Definition of combination operator" section). That is the case for (b) and (c), that include "minimize" and "maximize" respectively. For this specific geometry, (b) is highly compensated. Replacing the use of "minimize" by "avoid" would change the result as no compensation is allowed. This is illustrated by the ARs (d) and (f) for which, a zero local desirability automatically causes a zero global desirability.

Each AR's desirability is bounded by maximum and minimal values reported in Table 5. A set of rotations may produce conflicts between the action rules. For instance, at $\mathrm{Rx}=0^{\circ}$ and $\mathrm{Ry}=50^{\circ}$ the deformation risks, the overhanging non-machined surfaces and the datum surfaces return good desirability values $(0.717,0.893$ and 0.785 respectively), whereas all the other ARs have a poor desirability less than 0.3 (Fig. 17i). Another scenario at $\mathrm{Rx}=40^{\circ}$ and $\mathrm{Ry}=120^{\circ}$ would result in (b), (d), (e) and (f) greater than 0.7 and (a) and (c) are lower than average (Fig. 17ii).

For most of the scenarios, the rule (c) does not return a good desirability while the others are sufficient. In fact, the surfaces involved in this rule cannot be simultaneously vertical because of their perpendicularity. One maximum desirability of (c) is reached for $\mathrm{Rx}=90^{\circ}$ and $\mathrm{Ry}=0^{\circ}$, but the rules (d) and (f) would be minimal (Fig. 17iii). Several
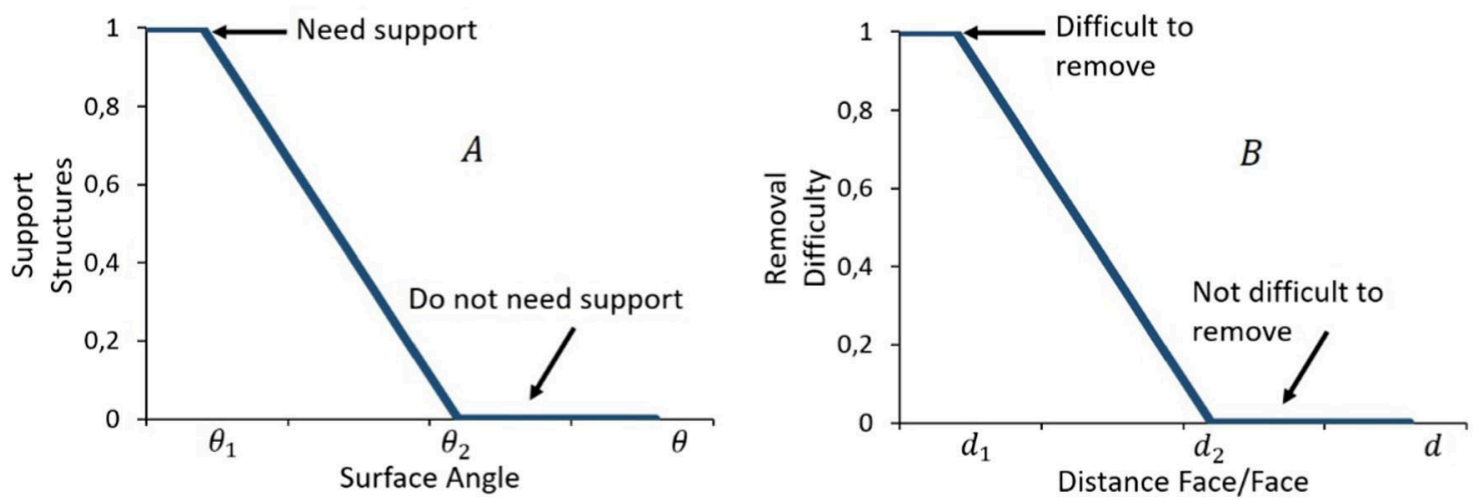

Fig. 13 Relational functions of surfaces with potential support difficult to remove: a support structure requirements; $\mathbf{b}$ support removal difficulty
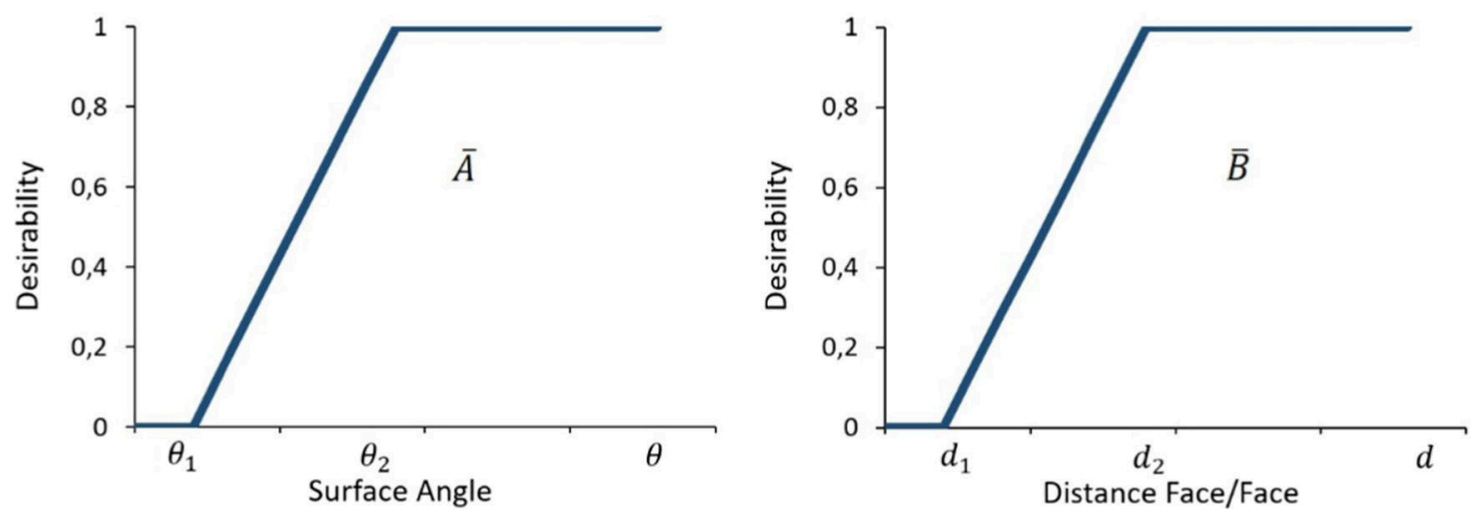

Fig. 14 Desirability functions of surfaces with potential support difficult to remove $(\bar{A})$ support structure requirements; $(\bar{B})$ support removal difficulty 

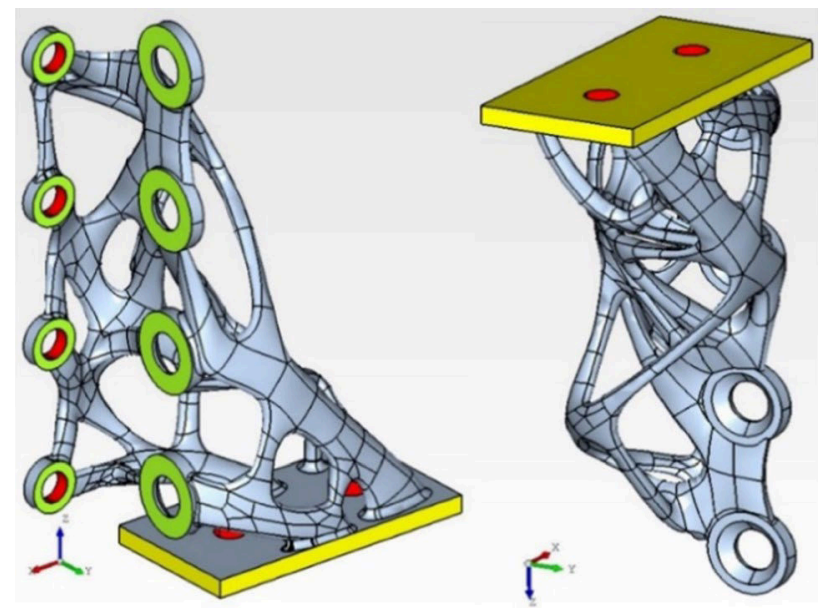

Fig. 15 Example of bracket

Table 4 Threshold values of example action rules

\begin{tabular}{ll}
\hline Action rules & Threshold values \\
\hline (a) & $A_{\text {smin }}=3813 \mathrm{~mm}^{2} ; A_{\text {smax }}=7737 \mathrm{~mm}^{2}$ \\
(b) & $\theta_{1}=30^{\circ} ; \theta_{2}=55^{\circ}$ \\
(c) & $\theta_{1}=45^{\circ} ; \theta_{2}=90^{\circ} ; \theta_{3}=135^{\circ}$ \\
(d) & $\theta_{1}=30^{\circ} ; \theta_{2}=55^{\circ} ; \theta_{3}=140^{\circ} ; \theta_{4}=160^{\circ}$ \\
(e) & $n_{1}=1 ; n_{2}=4$ \\
(f) & $\theta_{1}=30^{\circ} ; \theta_{2}=55^{\circ} ; d_{1}=1 \mathrm{~mm} ; d_{2}=35 \mathrm{~mm}$ \\
\hline
\end{tabular}

different other scenarios can also be analyzed. The one in Fig. 17iiii is an example that would be recommended for manufacturing the part in question. The rule (c) is not high, but is more than half of its maximum value.

Obviously, owing to this variety of solutions, it is not trivial to choose a point complying all of the AR simultaneously. However, one can make trade-offs by selecting a pair of angles for which the individual desirability reach an acceptable value. Alternatively, an aggregation of all of the responses as in the example of Eq. 1 can be useful to tackle this. One can also favor the use of optimization algorithms to find out constrained solutions. Extensive study aiming at proposing tools to facilitate the decision-making would be necessary.

As mentioned in the Introduction, action rules reflect in some ways criteria related to quality, cost and time. The quantitative evaluation enabled by the proposed knowledge modeling approach gives thus to the CAM user a big picture of how the extent of those criteria are respected.

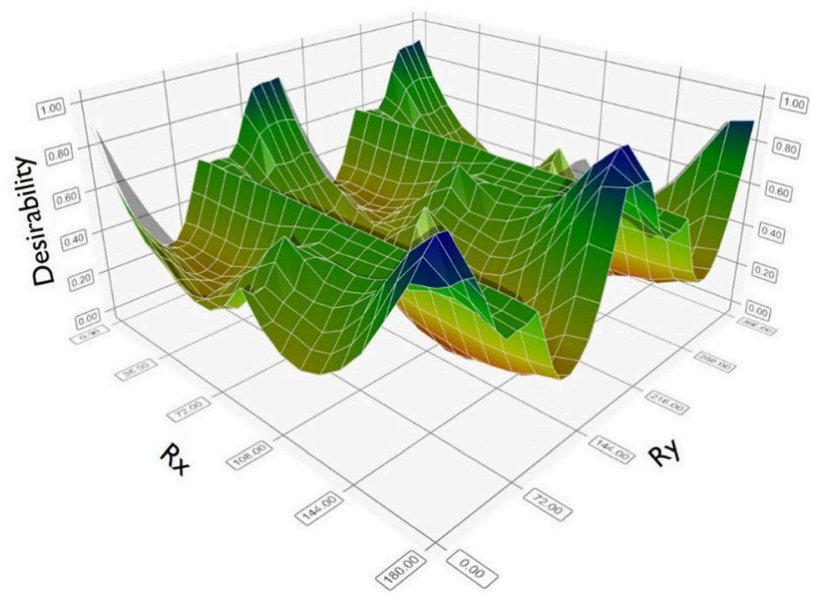

Fig. 16 Brackets resulting orientation desirability for part shadow action rule

Table 5 Minimum and maximum desirability values

\begin{tabular}{lll}
\hline Action rule & Minimum & Maximum \\
\hline (a) & 0.0 & 1.0 \\
(b) & 0.82 & 0.955 \\
(c) & 0.24 & 0.71 \\
(d) & 0.0 & 1.0 \\
(e) & 0.463 & 0.89 \\
(f) & 0.0 & 0.95 \\
\hline
\end{tabular}

\section{Conclusion}

This paper proposes a modeling approach to enable the transformation of elicitation data, more specifically the mathematical transformation of action rules into desirability functions. To achieve this quantitative representation process, evaluation concepts must be defined, calculated and combined thanks to the identification of variables and relational functions. In the framework of additive manufacturing, the elicitation of experts' knowledge has led to the identification of a list of action rules used by CAM engineers for part orientation, which is a key step in build preparation.

According to the modelling approach, in a first step each action rule is standardized through a simple grammar by the use of four action verbs (namely minimize, maximize, avoid, require, which explicitly show the objective of the rule) associated with an evaluation concept applied to some shape entities. Following that, a mathematization of the action rules content is done by performing a relational 
(i)

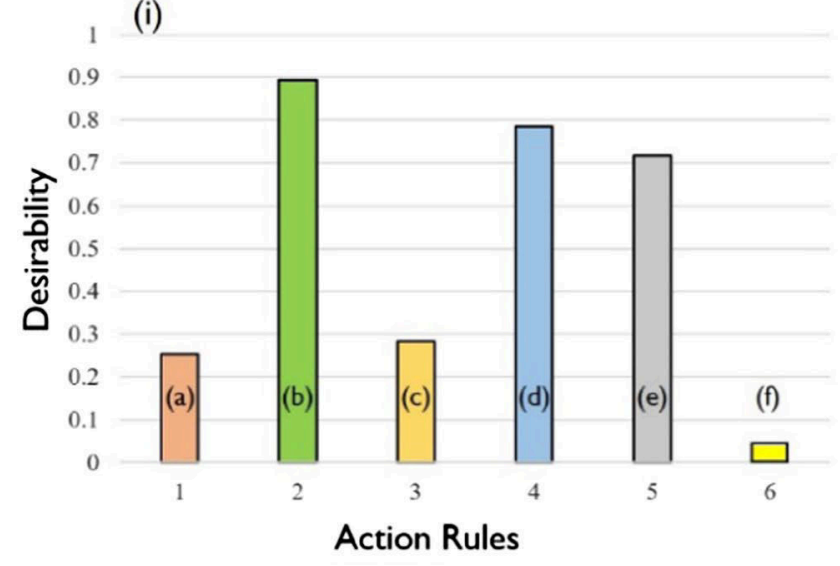

(iii)

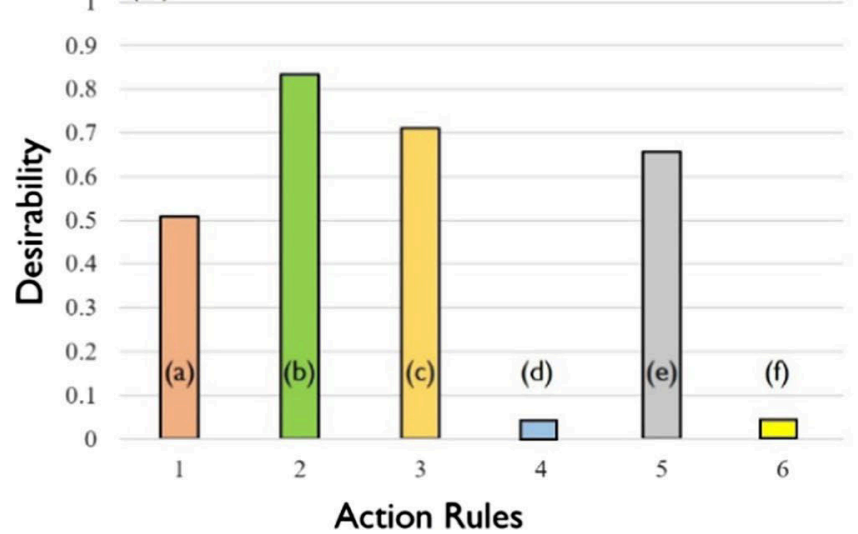

(ii)

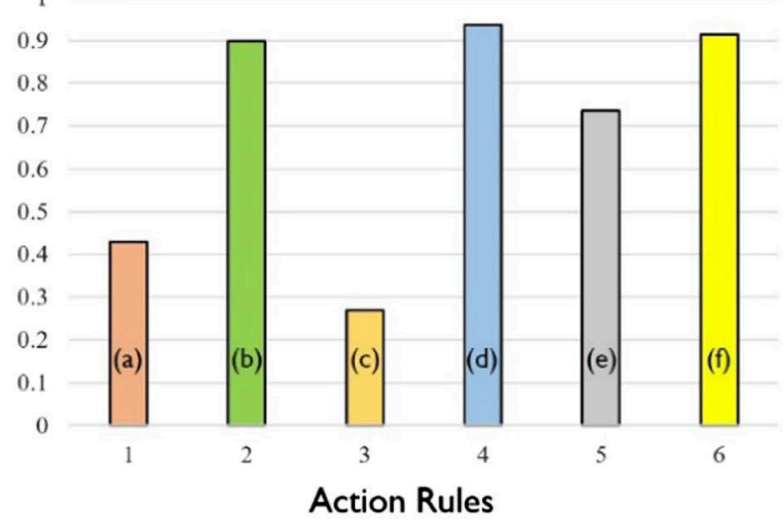

(iiii)

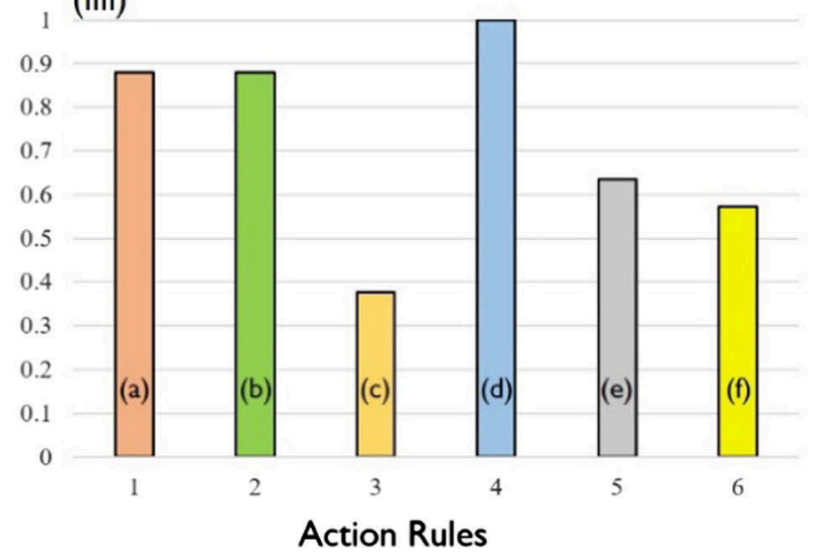

Fig. 17 Comparison of desirability values for the scenario: i $\mathrm{Rx}=0^{\circ} \mathrm{Ry}=50^{\circ}$; $\mathbf{i i} \mathrm{Rx}=40^{\circ} \mathrm{Ry}=120^{\circ}$; iii $\mathrm{Rx}=90^{\circ} \mathrm{Ry}=0^{\circ}$; iiii $\mathrm{Rx}=0^{\circ} \mathrm{Ry}=150^{\circ}$

analysis that provides the variable of the evaluation concept measurable from the shape. Then the mathematical expression of the evaluation concept is determined. The step of analyzing the relations between shapes entities and evaluation concept is complex; it requires the intervention of experts or a prior understanding of the domain of interest. The desirability function corresponding to each action rule is built based on the variable and the mathematical function. Through the modelling approach, mathematical considerations regarding the combination of multiple relational functions are provided.

As shown by the illustrative part, the present quantitative representation approach gives a big view of the satisfaction level of the expert rules. The results clearly show the robustness of the approach for which the aim is not to decide for the CAM operator but to assist his/her decision-making. Whilst the choice of an orientation is still manual, the orientation evaluation model is not arbitrary as it is based on desirability functions that represent experts' knowledge.
The way the CAM operator uses the proposed evaluation concepts to make its decision is of key importance. In future works, the way these results should be presented to the CAM operator to support his decision has to be studied. Several possibilities have already been proposed, such as:

- showing him the set of desirability side by side for all possible orientations (response surfaces),

- calculating and representing a weighted combination of the desirability values,

- limiting the orientation possibilities by selecting a threshold for each desirability.

The proposed mathematical models can also be integrated into optimization algorithms. How this multi-objective optimization can be done still have to be studied.

Another perspective would be to analyze how the orientation evaluation could influence the design of a shape through topology optimization. To perform shape optimization in a design process with this orientation evaluation used among 
other optimization criteria is also part of the project COFFA in which the current research has been conducted.

Acknowledgements This work benefited from the support of the Project COFFA ANR-17-CE10-0008 of the French National Research Agency (ANR) and DP Research Institute KAM Lab Project.

\section{Appendices}

\section{Definition 1 Negation}

This operation corresponds to the complementary of $f_{A}(\hat{y})$ defined as:

$\overline{f_{A}(\hat{y})}=1-f_{A}(\hat{y})$

Definition 2 Intersection

For a given scenario, the intersection $C$ (associated with $m_{c}$ ) of two relational functions $A$ and $B$ defined by the functions $f_{A}\left(\hat{y}_{A}\right)=m_{A}$ and $f_{B}\left(\hat{y}_{B}\right)=m_{B}$ respectively, corresponds to the logical "and" operation. Its corresponding function is given by:
$C=A \cap B$

$m_{c}=\operatorname{Min}\left[m_{A}, m_{B}\right]$

\section{Definition 3 Union}

For a given scenario, the union $C$ (associated with $m_{c}$ ) of $A$ and $B$ defined by the relational functions $f_{A}\left(\hat{y}_{A}\right)=m_{A}$ and $f_{B}\left(\hat{y}_{B}\right)=m_{B}$ respectively, is the logical "or" operation for which the corresponding function is:

$C=A \cup B$

$m_{C}=\operatorname{Max}\left[m_{A}, m_{B}\right]$

Definition 4 De Morgan Laws

$\overline{A \cup B}=\bar{A} \cap \bar{B}$

$\overline{A \cap B}=\bar{A} \cup \bar{B}$

Example of pseudocode for surface angle desirability computation:

Function SurfaceAngleDesi(ListOfFacet, DesiFunction, ActionType)

\{

TotalArea $=$ ListOfFacet.getTotalArea ()

for (int $\mathrm{i}=1 ; \mathrm{i}<$ ListOfFacet.size( $) ; \mathrm{i}++$ )

\{

angle $=$ ListOfFacet[i].getAngle( $)$

localDesi $=$ DesiFunction(angle)

area $=$ ListOfFacet[i].getArea ()

WeightedLocalDesi $=\operatorname{localDesi}^{\wedge}($ area/TotalArea $)$

\}

if ActionType $=$ Minimize or Maximize

return TotalDesirability $=$ Sum(WeightedLocalDesi $)$

else if ActionType $=$ Avoid or Require

return TotalDesirability $=$ Product(WeightedLocalDesi) 


\section{References}

Aggarwal, A., Singh, H., Kumar, P., \& Singh, M. (2008). Optimization of multiple quality characteristics for CNC turning under cryogenic cutting environment using desirability function. Journal of Materials Processing Technology, 205, 42-50.

Albert, M. (2001). Feature recognition-the missing link to automated CAM. Modern Machine Shop, 73(11), 70-78.

Al-Refaie, A., Al-Alaween, W., Diabat, A., \& Li, M.-H. (2017). Solving dynamic systems with multi-responses by integrating desirability function and data envelopment analysis. Journal of Intelligent Manufacturing, 28, 387-403.

Beissner, L., Jonassen, D. H., \& Grabowski, B. L. (1994). Using and selecting graphic techniques to aquire structural knowledge. Performance Improvement Quarterly, 7(4), 20-38.

Bullen, P. S., Mitrinovic, D. S., \& Vasic, M. (2003). Handbook of Means and their Inequalities. In Mathematics and its applications-Springer-Science + Business Media B.V, Chapter III.

Chergui, A., Hadj-Hamou, K., \& Vignat, F. (2018). Production scheduling and nesting in additive manufacturing. Computers \& Industrial Engineering, 126, 292-301.

CNRTL - CNRS French National Center for Textual and Lexical Resources. https://www.cnrtl.fr/definition/math\%C3\%A9mat isation.

Collins Dic. https://www.collinsdictionary.com/dictionary/english/ mathematization.

Cooke, N. J. (1994). Varieties of knowledge elicitation techniques. International Journal Human-Computer Studies, 41, 801-849.

Corney, J., Hayes, C., Sundararajan, V., \& Wright, P. (2005). The CAD/ CAM interface: a 25-year retrospective. Journal of Computing and Information Science in Engineering, 5(3), 188-197.

Costa, N. R., Lourenço, J., \& Pereira, Z. L. (2011). Desirability function approach: A review and performance evaluation in adverse conditions. Chemometrics and Intelligent Laboratory Systems, 107, 234-244.

Das, P., Chandran, R., Samant, R., \& Anand, S. (2015). Optimum part build orientation in additive manufacturing for minimizing part errors and support structures. Procedia Manufacturing, 1, 343-354.

Datta, S., Bandyopadhyay, A., \& Pal, P. K. (2006). Desirability function approach for solving multi-objective optimization problem in submerged arc welding. International Journal for Manufacturing Science and Production, 7(2), 127-138.

Davenport, T. H., De Long, D. W., \& Beers, M. C. (1998). Successful knowledge management projects. Sloan Management Review, $39(2), 43$.

de Jong, T., \& Ferguson-Hessler, M. G. M. (1996). Types and qualities of knowledge. Educational Psychologist, 31(2), 105-113.

de Pablos, P. O. (2002). Knowledge management and organizational learning: Typologies of knowledge strategies in the Spanish manufacturing industry from 1995 to 1999. Journal of Knowledge Management, 6(1), 52-62.

Delfs, P., Tows, M., \& Schmid, H.-J. (2016). Optimized build orientation of additive manufactured parts for improved surface quality and build time. Additive Manufacturing, 12, 314-320.

Derringer, G., \& Suich, R. (1980). Simultaneous optimization of several response variables. Journal of Quality Technology, 12(4), 214-219.

Frank, D., \& Fadel, G. (1995). Expert system-based selection of the preferred direction of build for rapid prototyping processes. Journal of Intelligent Manufacturing, 6, 339-345.
Ghaoui, S., Ledoux, Y., Vignat, F., Museau, M., Vo, T., Villeneuve, F., et al. (2020). Analysis of geometrical defects in overhang fabrications in electron beam melting based on thermomechanical simulations and experimental validations. Additive Manufacturing. https://doi.org/10.1016/j.addma.2020.101557.

Grandvallet, C., Mbow, M. M., Mainwaring, T., Vignat, F., Pourroy, F., \& Marin, P. R. (2020). Eight action rules for the orientation of additive manufacturing parts in powder bed fusion: An industry practice. International Journal on Interactive Design and Manufacturing, 14, 1159-1170.

Grandvallet, C., Pourroy, F., Prudhomme, G., \& Vignat, F. (2017). From elicitation to structuration of additive manufacturing knowledge. In 21st international conference on engineering design ICED17, Vol. 6: design information and knowledge, Vancouver, Canada 21-25.08.2017.

Gunasekaran, A., \& Ngai, E. W. T. (2007). Knowledge management in 21 st century manufacturing. International Journal of Production Research, 45(11), 2391-2418.

Gupta, B., Iyer, L., \& Aronson, J. (1999). An exploration of knowledge management techniques. In Association for information systems, AMCIS proceedings (p. 160).

Hussein, A., Hao, L., Yan, C., Everson, R., \& Young, P. (2013). Advanced lattice support structures for metal additive manufacturing. Journal of Materials Processing Technology, 213, 10191026. https://doi.org/10.1016/j.jmatprotec.2013.01.020.

Kim, K.-J., \& Lin, D. K. J. (2000). Simultaneous optimization of mechanical properties of steel by maximizing exponential desirability functions. Applied Statistics, 49(3), 311-325.

Kim, S., Rosen, D. W., Witherell, P., \& Ko, H. (2019). A design for additive manufacturing ontology to support manufacturability analysis. Journal of Computing and Information Science in Engineering. https://doi.org/10.1115/1.4043531.

Milton, N. R. (2007). Knowledge acquisition in practice: A step-bystep guide. Springer Science \& Business Media, ISBN 978-184628-861-6 (pp. 61-140).

Mourtzis, D., Makris, S., \& Chryssolouris, G. (2018). Computeraided manufacturing. In S. Chatti, L. Laperrière, G. Reinhart, \& T. Tolio (Eds.), CIRP encyclopedia of production engineering. Berlin: Springer. https://doi.org/10.1007/978-3-642-35950 -7_6550-4.

Nonaka, I., \& Takeuchi, H. (1995). The knowledge-creating company: How Japanese companies create the dynamics of innovation. London, New York: Oxford University Press. ISBN 0195092694.

Nonaka, I., \& Von Krogh, G. (2009). Perspective-Tacit knowledge and knowledge conversion: Controversy and advancement in organizational knowledge creation theory. Organization Science, 20(3), 635-652.

Paiva, E. L., Roth, A. V., \& Fensterseifer, J. E. (2002). Focusing information in manufacturing: A knowledge management perspective. Industrial Management and Data Systems, 102(7), 381-389.

Pandey, P. M., Reddy, N. V., \& Dhande, S. G. (2007). Part deposition orientation studies in layered manufacturing. Journal of Materials Processing Technology, 185, 125-131.

Qi, F., Mei, J.-Q., Xia, D.-F., \& Xu, S.-L. (2000). New proofs of weighted power mean inequalities and monotonicity for generalized weighted mean values. Mathematical Inequalities and Applications, 3(3), 377-383.

Qin, Y., Qi, Q., Scott, P. J., \& Jiang, X. (2019). Determination of optimal build orientation for additive manufacturing using Muirhead mean and prioritised average operators. Journal of Intelligent Manufacturing, 30, 3015-3034.

Rattanawong, W., Masood, S. H., \& Iovenitti, P. (2001). A volumetric approach to part-build orientations in rapid prototyping. Journal of Materials Processing Technology, 119, 348-353. 
Singh, A., Datta, S., Mahapatra, S. S., Singha, T., \& Majumdar, G. (2013). Optimization of bead geometry of submerged arc weld using fuzzy based desirability function approach. Journal of Intelligent Manufacturing, 24, 35-44.

Singh, M. D., Shankar, R., Narain, R., \& Kumar, A. (2006). Survey of knowledge management practices in Indian manufacturing industries. Journal of Knowledge Management, 10(6), 110-128.

Tan, L. P., \& Wong, K. Y. (2015). Linkage between knowledge management and manufacturing performance: A structural equation modeling approach. Journal of Knowledge Management, 19(4), 814-835.

Trautmann, H., \& Weihs, C. (2006). On the distribution of the desirability index using Harrington's desirability function. Metrika, 63, 207-213.

Vo, T. H., Museau, M., Vignat, F., Villeneuve, F., Ledoux, Y., \& Ballu, A. (2018). Typology of geometrical defects in electron beam melting. Procedia CIRP, 75, 92-97.

Wang, Y., Zheng, P., Xu, X., Yang, H., \& Zou, J. (2019). Production planning for cloud-based additive manufacturing-A computer vision-based approach. Robotics and Computer-Integrated Manufacturing, 58, 145-157.
Wilson, T. D. (2002). The nonsense of "knowledge management". Information Research, 8(1). http://informationr.net/ir/8-1/paper 144.html.

Zadeh, A. (1965). Fuzzy sets. Information and Control, 8, 338-353.

Zhang, Y., Bernard, A., Harik, R., \& Karunakaran, K. P. (2017). Build orientation optimization for multi-part production in additive manufacturing. Journal of Intelligent Manufacturing, 28, 1393-1407.

Zhou, K., Mo, L., Jin, J., \& Zain, A. M. (2019). An equivalent generating algorithm to model fuzzy Petri net for knowledge-based system. Journal of Intelligent Manufacturing, 30, 1831-1842.

Publisher's Note Springer Nature remains neutral with regard to jurisdictional claims in published maps and institutional affiliations. 